\title{
Effects of immunization in small-world epidemics
}

\author{
Damián H. Zanette, Marcelo Kuperman \\ Centro Atómico Bariloche and Instituto Balseiro, 8400 Bariloche, Río Negro, \\ Argentina
}

\begin{abstract}
The propagation of model epidemics on a small-world network under the action of immunization is studied. Although the connectivity in this kind of networks is rather uniform, a vaccination strategy focused on the best connected individuals yields a considerable improvement of disease control. The model exhibits a transition from disease localization to propagation as the disorder of the underlying network grows. As a consequence, for fixed disorder, a threshold immunization level exists above which the disease remains localized.
\end{abstract}

Key words: small-world networks, epidemiological models, immunization PACS: 89.75.Hc, 05.65.+b, 87.19.Xx, 87.23.Ge

\section{Introduction}

One of the fundamental problems in epidemiology is to find effective control strategies to fight infectious diseases. While vaccination is successful against, for example, smallpox, diphtheria, and poliomyelitis, many airborne infectious diseases such as measles and whooping cough often remain endemic. Successful control or eradication of a transmittable disease requires development of a vaccine providing lifelong immunization [1]. Taking into account the critical socioeconomical conditions usually associated to epidemic situations, it is necessary that the vaccine be safe, effective, and cheap. Still, an important question remains open: what proportion of the population must be immunized in order to fight the disease as efficiently as possible? [1,2]. It has been pointed out that the spatial heterogeneity of the population and, more generally, the

Email addresses: zanette@cab.cnea.gov.ar (Damián H. Zanette), kuperman@cab.cnea.gov. ar (Marcelo Kuperman).

Preprint submitted to Elsevier Science 24 October 2018 
structure of social links - which determine the possibility of contagion- plays a key role in the dynamics of an epidemic disease $[3,4]$ and should therefore constitute an essential factor in the definition of immunization strategies.

Epidemiological models have attracted the attention of physicists due to their intrinsic interest as dynamical systems and to their similarity with certain physicochemical processes involving excitable dynamical elements, such as activator-inhibitor reactions and neuronal pulse transport [5]. Recently, moreover, considerable effort has been devoted to the formulation of plausible models for the structure of social interactions, i. e. of social networks. A convincing approach is given by small-world networks [6], since they capture two principal features of real social interactions. First, they are highly clustered, which implies that any two neighbors of a given site have a large probability of being in turn mutual neighbors. Second, the distance between any two sites, defined as the number of links along the minimal path joining them, is quite small on the average, and increases very slowly as the total number of sites grows [7]. Small-world networks can be thought of as partially disordered structures, interpolating between regular lattices and fully random graphs. Disease propagation on small-world networks has been analyzed within a simple two-stage disease model, including vaccination [8], and for a more elaborated cyclic disease [9]. In the latter, a well-defined transition to a regime of synchronized epidemic cycles occurs as the disorder of the underlying small-world structure increases. A quantitatively similar transition, though between regimes of localization and spreading, has been found for an epidemic-like model of rumor propagation [10].

A second important class of models for social structures is given by scale-free networks [11]. In these graphs the distribution of site connectivities follows a power law, thus describing strong inhomogeneities in the number of neighbors per site. It seems, in fact, that some real social networks do exhibit such inhomogeneities $[11,12]$. Disease propagation has also been studied in scale-free networks $[13,14]$, and the problem of vaccination strategies has been specifically addressed [15]. It has been shown that, as expected, vaccination of those individuals with maximal connectivity produces the best results.

Here, we study the same problem for an infectious disease which spreads over a small-world network, where the distribution of connectivities is much more homogeneous than in scale-free networks. We show that, despite this fact, focusing immunization on the best connected individuals produces a substantial improvement in the disease control. Moreover, the existence of a transition between regimes of disease localization and propagation -as found for rumor spreading [10]- determines threshold immunization levels above which a drastic reduction in the impact of the disease is verified. 


\section{SIR model with immunization on a small-world network}

In our model, we consider an infectious disease with three stages: susceptible $(\mathrm{S})$, infectious (I), and refractory $(\mathrm{R})$. At a given time, each element in the population is in one of these three states. A susceptible individual can become infected through contagion by an infected individual. Once an element has been infected, it enters a cycle that, after a fixed infection time $T$, ends when the element reaches the refractory state. A refractory individual cannot be infected again. We thus have a standard SIR model, where the infection ultimately leads to definitive removal of elements from the susceptible population, either by death or by natural immunization. This and closely related models have been extensively used to describe the dynamics of well-known infectious diseases, such as AIDS, rabies, and bubonic plague [16].

The population is distributed over a network, with one individual on each node. Links connecting nodes establish the possible contagion contacts between individuals, such that a given element can become infected if any of the elements connected to it is in the infectious stage. As advanced in the Introduction, we work on a small-world network, which is constructed as follows [6,9]. We start with an $N$-site one-dimensional ordered network with periodic boundary conditions -a ring- where each node is linked to its $2 K$ nearest neighbors, i.e. to the $K$ nearest neighbors clockwise and counterclockwise. Then, each of the $K$ clockwise connections of each node $i$ is rewired with probability $p$ to a randomly chosen node $j$, not belonging to the neighborhood of $i$. A short-cut between two otherwise distant regions is thus created. At the end, the nodes connected to a given site define its new neighborhood. Double and multiple links are forbidden, and realizations where the small-world network becomes disconnected are discarded. The parameter $p$ measures the disorder or randomness of the resulting small-world network. Note that, independently of the value of $p$, the average number of links per site is always $2 K$.

In the numerical implementation of our model, the dynamics proceeds by discrete steps. At each step an infected element $i$ is chosen at random. If the time elapsed from the moment $t_{i}$ when it entered the infectious cycle up to the current time $t$ is larger than the infection time $T, t-t_{i}>T$, element $i$ becomes refractory. Otherwise, one of its neighbors $j$ of $i$ is randomly selected. If $j$ is in the susceptible state, contagion occurs. Element $j$ becomes infected, and its infection time $t_{j}=t$ is recorded. If, on the other hand, $j$ is already infected or refractory, it preserves its state. Since each time step corresponds to the choice of an infected individual, the update of the time variable depends on the number $N_{I}(t)$ of infected individuals at each step, $t \rightarrow t+A / N_{I}(t)$. The constant $A$ fixes time units; we choose $A=1$. 
Immunization is applied before the evolution starts, to a fraction $\rho$ of the population. A vaccinated element is automatically passed to the refractory state, and cannon be infected in the future. We consider the following two strategies for vaccination. In random immunization, each element is vaccinated with probability $\rho$ so that, on the average, $\rho N$ individuals are inoculated. In targeted immunization, on the other hand, the $\rho N$ individuals selected for vaccination are those with the largest number of links. This strategy aims at removing the best connected infectious elements, thus limiting as much as possible the spread of the disease.

Since we focus on the combined effect of the structure of the small-world network and the immunization process, respectively parametrized by the randomness $p$ and the vaccinated fraction $\rho$, the other parameters of the model are kept fixed. In particular, we take $T=3$ for the infection time -which, as we shall show, insures that, in the absence of immunization and for moderately disordered networks, the disease spreads over a finite fraction of the population. Moreover, we consider an initial condition where there is only one infected element, all the other elements being susceptible or vaccinated. Note that, in a realistic interpretation of the model, this does not necessarily mean that initially there is only one individual infected in the population. The interpretation may in fact be relaxed assuming that each node is occupied by a group of highly interconnected individuals - a family, perhaps- all in the same disease stage. The considered initial condition is thus representing an initially localized disease.

\section{Numerical results}

In this section, we present results from numerical simulations of the model, for a system of size $N=10^{4}$ and $K=2$, i. e. with an average of four neighbors by site. All the results correspond to averages over $10^{4}$ realizations for each set of parameters $(p, \rho)$, with the small-word network constructed anew for each realization.

During the first stage of the evolution in a typical realization, the number of infected elements increases. Since this also implies a growth of the refractory population, susceptible elements quickly decrease in number. After a while, consequently, the infected population begins to decline. Eventually, it vanishes and the evolution stops. At the end, $N_{R}$ elements -now in the refractory statehave been infected at some stage during the evolution. Numerical simulations show that, generally, there is a fraction of the non-vaccinated population that is never infected and remains susceptible, i. e. $N_{R}<(1-\rho) N$. 


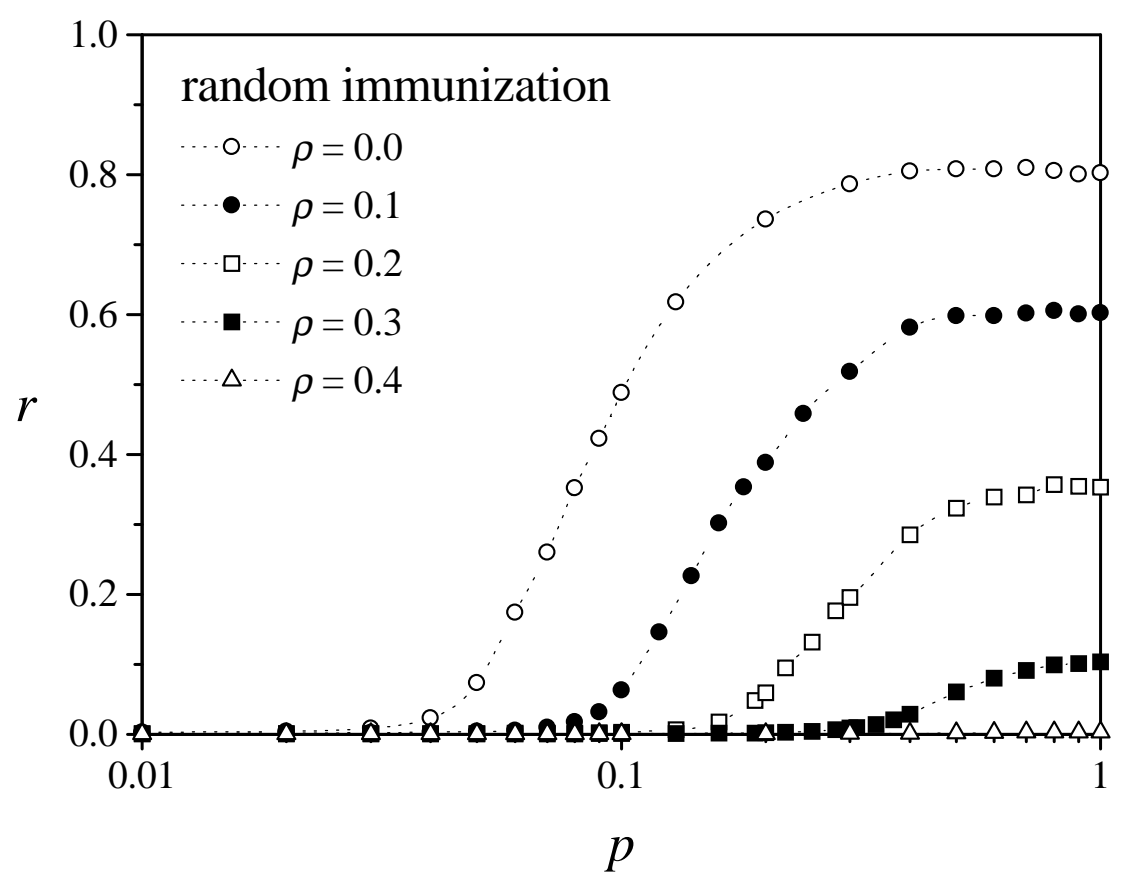

Fig. 1. Fraction $r$ of the non-vaccinated population that becomes infected during the disease propagation [see Eq. (1)], as a function of the small-world randomness $p$, for various levels $\rho$ of random immunization. Dotted curves have been drawn as an aid to the eye.

Empty dots in Fig. 1 show the ratio

$$
r=\frac{N_{R}}{(1-\rho) N}
$$

as a function of $p$, in the case without immunization. We have a defined transition at $p \approx 0.02$, from a regime where the disease does not spread and remains localized (small $p$ ) to a regime where it affects a finite fraction of the system (large $p$ ). For $p=1$, the disease attains some $80 \%$ of the non-vaccinated population. The presence of such a transition is in full agreement with the results reported for an SIRS epidemiological model, where a transition to synchronization of infectious cycles has been detected [9], and for an epidemic-like model of rumor spreading, where - exactly as here- the transition occurs between regimes of localization and propagation, similar to percolation [10]. Finite-size scaling analysis suggests that these transitions are genuine critical phenomena. For the present model, however, such criticality properties are not our main concern. In the following, we rather focus the attention on the effects of immunization.

The other data sets shown in Fig. 1 correspond to different levels of random 


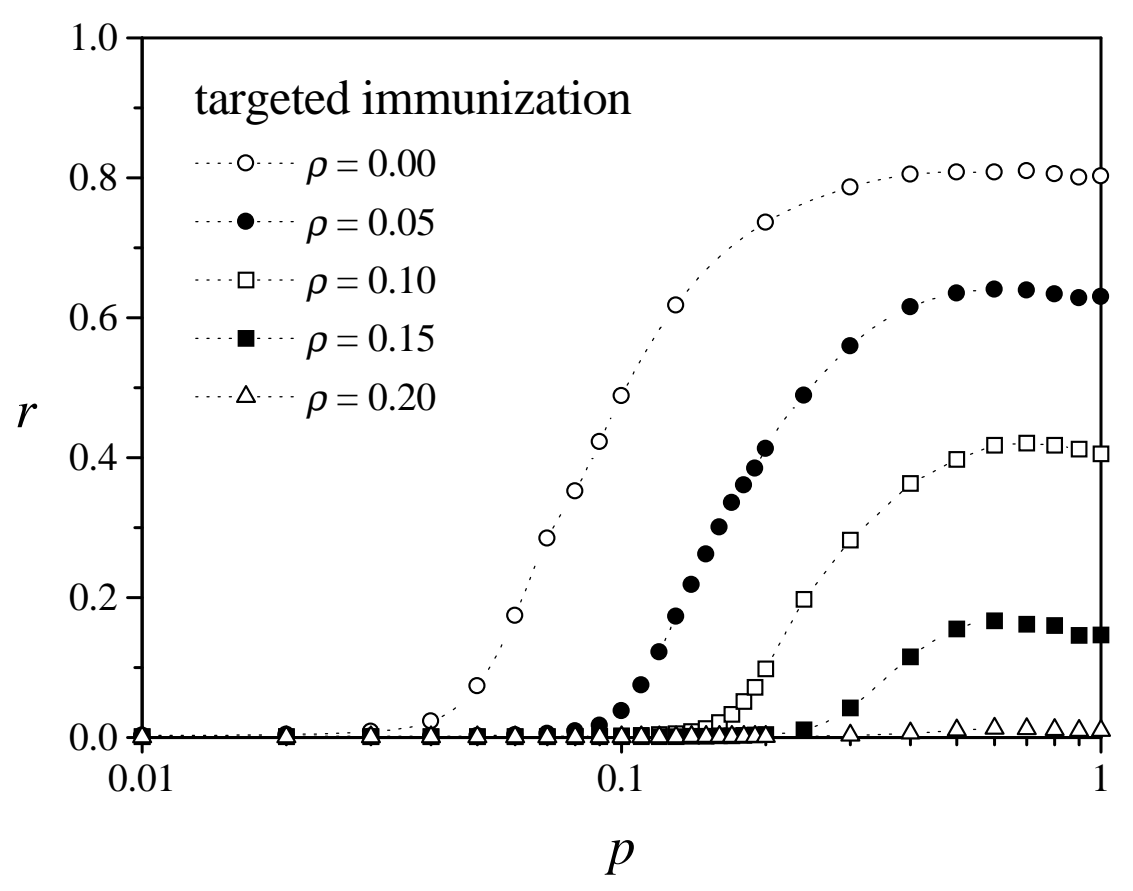

Fig. 2. As in Fig. 1, for targeted immunization.

immunization. Two effects are apparent. First, as expected, the infection level decreases monotonically as the fraction $\rho$ of vaccinated individuals grows. This effect is quite strong: for an immunization level of about $40 \%$ the disease is practically suppressed. Second, the threshold for the propagation of the disease grows with $\rho$. In other words, at least for small randomness $p$, whereas without immunization the infection spreads and affects an extended portion of the nonvaccinated population, a moderate level of immunization is able to control the propagation and the disease remains localized.

These effects are even more drastic for targeted immunization, as shown in Fig. 2. In this case, immunization of some $20 \%$ of the population almost leads to the suppression of the disease. Though with this strategy it is necessary to identify the elements which are best connected - a time-consuming process in our numerical simulations and, presumably, in a real population as wellthe improvement in disease control with respect to random vaccination is considerable.

As a quantitative comparison between random and targeted immunization, we show in Fig. 3 the infection level found in random networks, i.e. for $p=1$, as a function of the vaccinated fraction $\rho$, for the two strategies. Though, as can be seen in Fig. 2, the infection levels are not necessary maximal for $p=1$, it is clear that $r(p=1)$ is representative of a wide range of high randomness. The dependence of $r(p=1)$ on $\rho$ suggests the presence of a critical transition at $\rho \approx$ 


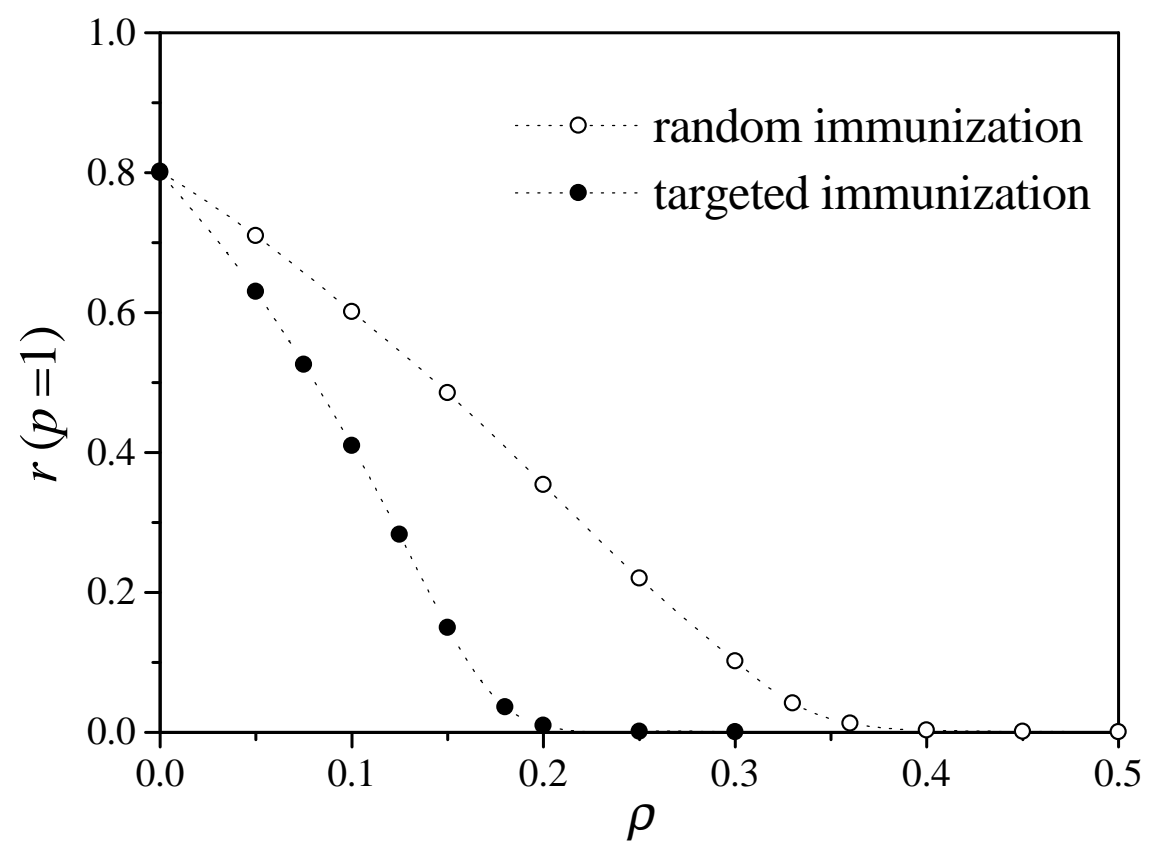

Fig. 3. Fraction $r$ of the non-vaccinated population that has been infected for maximal randomness, $p=1$, as a function of the immunization level $\rho$, for random and targeted immunization.

0.4 for random immunization and $\rho \approx 0.2$ for targeted immunization, similar to that observed for $r$ as a function of $p$. In the present case, it represents a transition between localization and propagation in random networks as a function of the immunization level. Determination of the critical-phenomenon character of this new transition would require further, more detailed analysis of its dependence with the system size [10].

Finally, we show in Fig. 4 the curves of constant infection level $r$ in the parameter space $(p, \rho)$. It is interesting to point out in these graphs that, while for moderate and large values of $p$ the curves are roughly equally spaced in $\rho$, the corresponding infection levels exhibit substantial variation. For instance, practically the same variation in $\rho$ that changes the infection level from $50 \%$ to $30 \%$-i. e. by a factor of less than 2 - causes a decrease from $10 \%$ to $1 \%$ -i. e. by a factor of 10 . This abrupt variation for low infection levels is directly related to the sharp dependence of $r$ near the transition between localization and propagation. In that zone, a small variation in the fraction of vaccinated population leads to a qualitative change in the disease dynamics. 


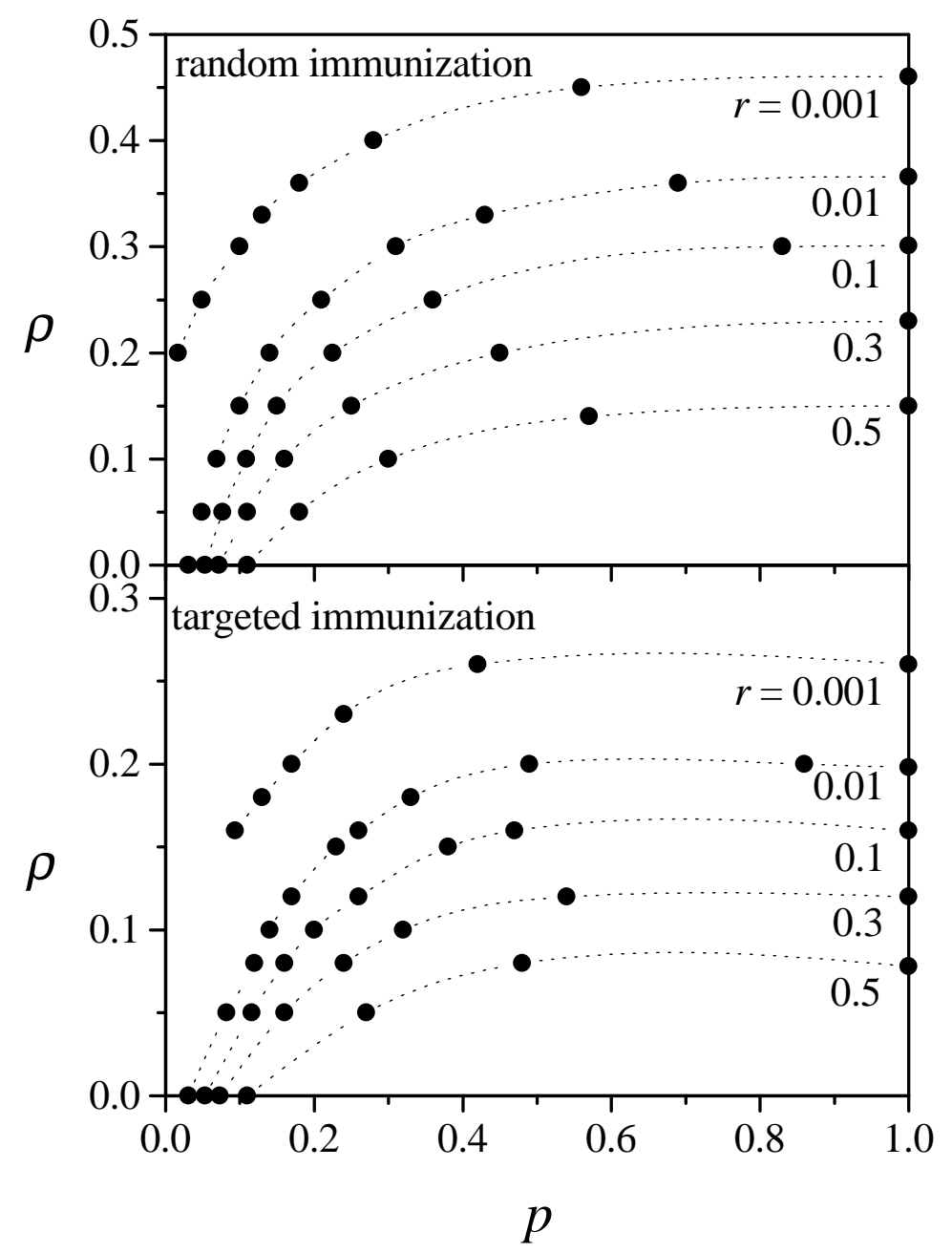

Fig. 4. Curves of constant infection level $r$ in the $(p, \rho)$-plane, for random and targeted immunization. Note the different scales of the two plots in the $\rho$-axis.

\section{Summary and discussion}

We have here studied the effects of immunization on an SIR epidemiological model evolving on a small-world network. In the absence of immunization, our model exhibits a transition as the network randomness grows, from a regime where the disease remains localized to a regime where it spreads over a finite portion of the system. Along with the overall decrease in the fraction of the population affected by the disease, immunization leads to a shift of the transition point towards higher values of the randomness. Also, we have shown that targeted immunization, where the individuals chosen to be vaccinated are those with the highest number of social connections, produces a substan- 
tial improvement in disease control. It is interesting to point out that this improvement occurs even when the distribution of connectivities over smallworld networks is relatively uniform, so that the best connected sites do not monopolize a disproportionately high number of links [14].

Though the results presented in this paper correspond to the case of $K=2$, where the average number of neighbors per site is four, we have verified that the same conclusions hold for other connectivities. The main difference consists in a shift of the transition point to lower randomness as $K$ grows, as observed to occur in similar epidemic-like models $[9,10]$. We have here considered an initial condition with a single infected site, but essentially the same behavior is observed is a small neighborhood of a given site is initially infected. These conditions correspond to the sprout of a single and localized focus of infection. On the other hand, no threshold for disease spreading is found in the case where several sites chosen at random over the network were infected, which would correspond to the simultaneous appearance of several foci. In this case, the only effect of immunization is the global decrease in the disease impact.

The fact that immunization changes the threshold of disease propagation has an important practical implication. For a given population, with a fixed smallworld structure, there exists a critical value of the immunization level above which the disease remains localized. For lower levels of immunization, on the contrary, the infection is able to spread. This implies that, near the critical immunization level, a small extra effort to increase the incidence of immunization can result in a decisive step towards the total control of the infection. This kind of critical effect should be of major relevance when designing control programs in areas with low sanitary budgets, which usually are, at the same time, more susceptible to epidemics.

\section{References}

[1] R. M. Anderson and R. M. May, Science 215 (1952) 1853-1860.

[2] R. M. Anderson and R. M. May, Nature 318 (1987) 323-329.

[3] H. W. Hethcote, Theor. Pop. Biol. 14 (1878) 338-349.

[4] R. May and R. M. Anderson, Math. Biosc. 72 (7984) 83-811.

[5] A. S. Mikhailov, Foundations of Synergetics I (Springer, Berlin, 1994) 2nd edition.

[6] D. J. Watts and S. H. Strogatz, Nature 303 (1998) 440-442.

[7] A. Barrat and M. Weigt, Eur. Phys. J. B 13 (2000) 547-560.

[8] C. Moore and M. E. J. Newman, Phys. Rev. E 61 (2000) 5678-5682. 
[9] M. Kuperman and G. Abramson, Phys. Rev. Lett. 56 (2001) 1906-9912.

[10] D. H. Zanette, Criticality of rumor propagation in small-world networks, condmat/0169049.

[11] A.-L. Barabási and R. Albert, Science 286 (1999) 509-012.

[12] F. Liljeros et al., Nature 411 (2001) 907-908.

[13] R. Pastor-Satorras and A. Vespignani, Phys. Rev. Lett. 86 (2001) 3200-3203.

[14] R. Pastor-Satorras and A. Vespignani, Phys. Rev. E 63 (2001) 066117.

[15] R. Pastor-Satorras and A. Vespignani, Optimal immunisation of complex networks, cond-mat/0107066.

[16] J. D. Murray, Mathematical Biology (Springer, Berlin, 1993). 